EXTENDED REPORT

\title{
Central corneal thickness and progression of the visual field and optic disc in glaucoma
}

\author{
B C Chauhan, D M Hutchison, R P LeBlanc, P H Artes, M T Nicolela
}

Br J Ophthalmol 2005;89:1008-1012. doi: 10.1136/bjo.2004.062927

See end of article for authors' affiliations .....................

Correspondence to: Balwantray C Chauhan, Department of Ophthalmology and Visual Sciences, Dalhousie University, 2nd Floor Centennial Building, Queen Elizabeth II Health Sciences Centre, Halifax, NS, Canada B3H 2Y9; bal@dal.ca

Accepted for publication 1 February 2005

\begin{abstract}
Aims: To determine whether central corneal thickness (CCT) is a significant predictor of visual field and optic disc progression in open angle glaucoma.

Methods: Data were obtained from a prospective study of glaucoma patients tested with static automated perimetry and confocal scanning laser tomography every 6 months. Progression was determined using a trend based approach called evidence of change (EOC) analysis in which sectoral ordinal scores based on the significance of regression coefficients of visual field pattern deviation and neuroretinal rim area over time are summed. Visual field progression was also determined using the event based glaucoma change probability (GCP) analysis using both total and pattern deviation.

Results: The sample contained 101 eyes of 54 patients (mean (SD) age 56.5 (9.8) years) with a mean follow up of $9.2(0.7)$ years and 20.7 (2.3) sets of examinations every 6 months. Lower CCT was associated with worse baseline visual fields and lower mean IOP in the follow up. In the longitudinal analysis CCT was not correlated with the EOC scores for visual field or optic disc change. In the GCP analyses, there was a tendency for groups classified as progressing to have lower CCT compared to nonprogressing groups. In a multivariate analyses accounting for IOP, the opposite was found, whereby higher CCT was associated with visual field progression. None of the independent factors were predictive of optic disc progression.

Conclusions: In this cohort of patients with established glaucoma, CCT was not a useful index in the risk assessment of visual field and optic disc progression.
\end{abstract}

\section{METHODS \\ Patients}

Patients in this study were in a longitudinal prospective study on psychophysical tests and scanning laser tomography in open angle glaucoma. They were recruited consecutively from the glaucoma clinics of our institution with the following inclusion criteria: (i) diagnosis of open angle glaucoma with obvious notching or progressive thinning of the neuroretinal rim, typically recorded photographically; (ii) visual field with a mean deviation index between $-2 \mathrm{~dB}$ and $-10 \mathrm{~dB}$; (iii) open angles by gonioscopy, and (iv) best corrected visual acuity $\geqslant 6 / 12$. Exclusion criteria were: (i) concomitant ocular disease; (ii) systemic disease or systemic medication known to affect the visual field; (iii) refractive error exceeding $5 \mathrm{D}$ equivalent sphere or $3 \mathrm{D}$ of astigmatism; and (iv) contact lens wear.

\section{Testing protocol}

Both eyes of patients were tested every 6 months after enrolment. Visual field examinations were carried out with static automated perimetry using the full threshold 30-2 program of the Humphrey field analyser (Carl Zeiss Meditech, Dublin, CA, USA). Scanning laser tomography was performed with the Heidelberg retina tomograph (Heidelberg Engineering GmbH, Dossenheim, Germany). At each session, several images with the optic disc centred in the image frame were obtained. Three of the best quality images were used to compute the mean topography image. A contour line was carefully drawn and imported to the follow up

Abbreviations: CCT, central corneal thickness; EOC, evidence of change; GCP, glaucoma change probability; MD, mean deviation; OHTS, Ocular Hypertension Treatment Study; PSD, pattern standard deviation; RA, rim area 
images for analysis using the Heidelberg Eye Explorer software ( $\mathrm{v}$ 1.4.0.1). CCT was measured in one session using an ultrasound pachymeter (DGH-550 Pachette 2, DGH Technology Inc, Exton, PA, USA) within 6 months of the end of the follow up. Both perimetry and scanning laser tomography were carried out at the same visit throughout the follow up.

\section{Data analysis}

The visual field and optic disc data were analysed in an identical manner using a recently devised trend analysis called "evidence of change" (EOC) analysis. The technique has been described fully elsewhere ${ }^{14}$ but, briefly, for the visual field, ordinal scores are given to each of four sectors (superior, inferior, temporal, and central as described by Junemann ${ }^{15}$ ) depending on the significance of the regression of the mean pointwise pattern deviation value over follow up time. An integer EOC score ranging from 0 (where the regression is non-significant, $\mathrm{p}>0.1$ ) to 5 (where the regression is highly significant, $p<0.002$ ) is given to each sector. Similarly, the optic disc was divided into four sectors corresponding to the visual field (inferior, superior, nasal, and temporal, respectively ${ }^{15}$ ). The sectoral optic disc EOC score was derived in an identical manner to that of the visual field based on the regression of sectoral neuroretinal rim area over follow up time. The summed EOC scores therefore ranged from 0 (very little evidence of change) to 20 (extremely high evidence of change). This analysis approach has several advantages among which is the non-reliance on a single cut-off value in slope significance, but provision of a weighted score over the range of significance values, from borderline to high significant.

The visual field was also analysed using the glaucoma change probability (GCP) analysis, ${ }^{16}$ where test points in follow up examinations that are outside of the retest variability limits are indicated as black triangles, suggestive of deterioration. We used customised software to identify the maximum number of overlapping black triangles in three consecutive follow up examinations based on both total deviation and pattern deviation.

Spearman rank order correlation coefficients were calculated for the associative analysis involving EOC scores. For all other correlations, Pearson's correlation coefficients were used. The sample was divided into approximately equal thirds based on CCT for a comparison of the mean groupwise EOC scores for the visual field and optic disc, as well as the maximum number of black triangles for both total and pattern deviation analyses. Slopes of mean deviation (MD) and neuroretinal rim area (RA) were computed and compared across the CCT groups using a Kruskal-Wallis test. For multivariate analysis of visual field and optic disc change, we used baseline MD, pattern standard deviation (PSD), mean and SD of IOP during the follow up, and CCT as independent variables. All tests were two tailed and statistical significance was assumed when $\mathrm{p}<0.05$.

\section{RESULTS}

Our sample contained 101 eyes of 54 patients whose mean (SD) age at baseline was 56.5 (9.8) years. There were 29 (54\%) men and 25 (46\%) women, of whom 52 (96\%) were European derived and two (4\%) were African derived. The mean follow up was 9.2 (0.7) years with a mean of 20.7 (2.3) sets of 6 monthly visual field and optic disc examinations. The overall mean CCT was 547 (41) $\mu \mathrm{m}$, while that of the three subgroups $(<524 \mu \mathrm{m}, 524-567 \mu \mathrm{m}$, and $>567 \mu \mathrm{m})$ was 504 (17) $\mu \mathrm{m}(\mathrm{n}=35), 544$ (13) $\mu \mathrm{m}(\mathrm{n}=33)$, and 594 (19) $\mu \mathrm{m}$ $(\mathrm{n}=33)$ respectively.

In a univariate analyses, there were statistically significant relations between CCT and baseline MD ( $r=0.254$, $\mathrm{p}=0.010)$ and PSD $(r=-0.313, \mathrm{p}=0.001)$, indicating that lower CCT was associated with worse baseline visual fields. Higher CCT was associated with higher mean IOP during the follow up $(r=0.264, \mathrm{p}=0.015)$, but not with maximum IOP, its SD during follow up, or refractive error $(\mathrm{p}>0.350)$. In a multivariate analysis, none of these independent variables reached statistical significance $(\mathrm{p}>0.150)$.

The relation between CCT and the EOC scores for both the visual field and optic disc was poor $(r=-0.133, \mathrm{p}=0.184$; and $r=0.074, \mathrm{p}=0.483$, respectively; fig $\mathrm{l}$, data points coded according to mean IOP in the follow up). The correlation analysis was repeated for the maximum number of overlapping black triangles in the GCP analysis. There was a large range in visual field progression; however, in this different form of analysis, visual field progression was not also related to CCT $(r=0.072, \mathrm{p}=0.474$ for the total deviation analysis and $r=0.031, \mathrm{p}=0.760$ for the pattern deviation analysis; fig 2).

The sample was divided into visual field progressing and non-progressing groups based on the number of overlapping black triangles in three consecutive examinations. A cut-off criterion of two, three, and four black triangles was used for both the total and pattern deviation analysis. For both forms of analysis, there was a tendency for the progressing patients to have a lower CCT than progressing ones (fig 3); however, the difference diminished with increasingly conservative criteria. Only the criterion of two overlapping black triangles in the pattern deviation analysis yielded a statistically significant difference in CCT between the progressing and
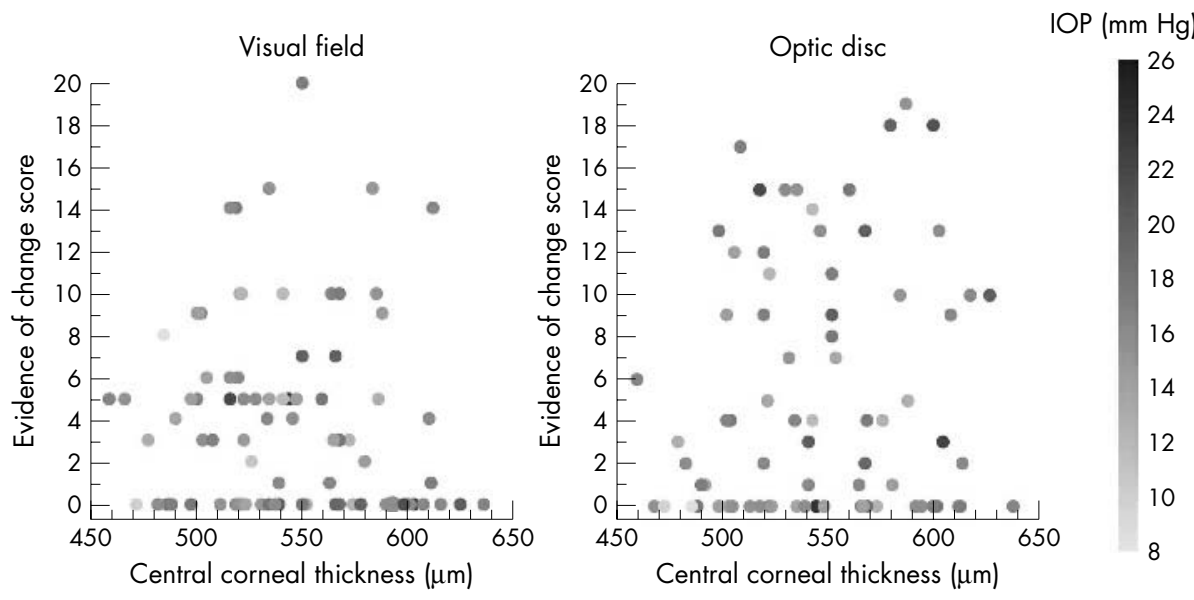

Figure 1 Relation between evidence of change score for pattern deviation (left) and neuroretinal rim area (right) and central corneal thickness. Data points are coded according to mean intraocular pressure (IOP) in the follow up. 

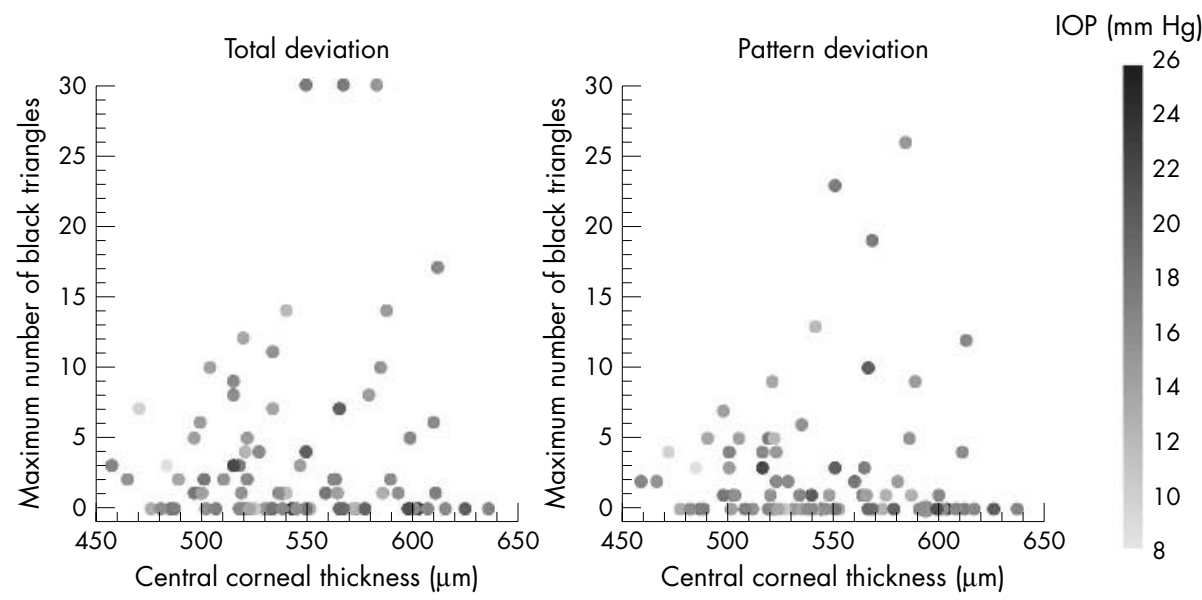

Figure 2 Relation between the maximum number of overlapping black triangles in three consecutive tests in the glaucoma change probability analysis based on total deviation (left) and pattern deviation (right) and central corneal thickness. Data points are coded according to mean intraocular pressure (IOP) in the follow up.

non-progressing groups (mean 535 (42) $\mu \mathrm{m}$ and 553 (39) $\mu \mathrm{m}$, respectively; $\mathrm{p}=0.039$ ).

There were no statistically significant differences ( $p>0.150$, analysis of variance) in either of the four indices of change in the visual field and optic disc between groups stratified according to CCT (table 1). The data indicate widely overlapping values in the indices of change among the three groups. MD slope and CCT were poorly correlated $(r=-0.020, \quad \mathrm{p}=0.842)$ as were RA slope and CCT $(r=0.004, \mathrm{p}=0.967)$. These slopes were not significantly different across the three CCT groups $(\mathrm{p}>0.295)$.

In the multivariate analyses of the EOC scores and the glaucoma change probability analyses, baseline PSD and the SD of IOP during the follow up were significant for all three methods of visual field analyses (table 2 ). In all cases the regression coefficients were positive indicating an association between visual field progression and higher baseline PSD and SD of IOP. CCT was statistically significant only for the total deviation analysis and borderline significant for the pattern deviation analysis. In both cases, the coefficients indicated a positive relation-that is, higher CCT was related to greater visual field progression.

\section{DISCUSSION}

The cornea and sclera form the continuous collagenous external coat of the eye. In the posterior segment the sclera perforates to form the lamina cribrosa through which retinal ganglion cell axons exit the eye. Changes in the sclera may be highly relevant in glaucoma, and scleral properties, such as elasticity and thickness may mirror those in the lamina. In experimental glaucoma there is acquired regional thinning in the posterior sclera that increases eye wall stress, ${ }^{17}$ suggesting that individuals with thinner scleras may be at a higher risk from glaucomatous damage. There is also acquired scleral thinning in human $^{18}$ and experimental myopia, ${ }^{19}$ and strong epidemiological evidence that increasingly high myopia is associated with increasing prevalence of glaucoma. $^{21}$ Finally, recent evidence indicates that laminar thickness is reduced in highly myopic eyes, and even more in highly myopic glaucomatous eyes. ${ }^{22}$ While the relation between scleral changes and glaucoma is not fully understood, there is compelling evidence suggesting that posterior scleral and laminar thickness may be useful indices in the clinical assessment of glaucoma. To date, however, there are no clinically available methods to measure posterior scleral or laminar thickness, but because of the close relation between the sclera and lamina, and by implication the lamina and cornea, many investigators have focused on the role of CCT in glaucoma. While CCT is known to confound the clinical estimate of IOP, ${ }^{23}$ thus far its role, after accounting for IOP effects, is not clear.

This study demonstrates the lack of a consistent relation between CCT and visual field or optic disc progression in both univariate analysis and multivariate analysis, in which the effects of IOP were accounted for. While patients classified as progressing with three increasingly conservative GCP criteria tended to have lower mean CCT, in the multivariate analysis there was a tendency for the opposite-that is, visual field progression was associated with higher CCT. Hence, in spite of the suggestive relation between CCT and visual field progression, the associations were contradictory. In contrast, progressive visual field damage was consistently associated with a higher baseline PSD and higher SD of IOP during the follow up, the latter being a finding that has been reported previously. ${ }^{23-25}$ Patients showing clinical progression would

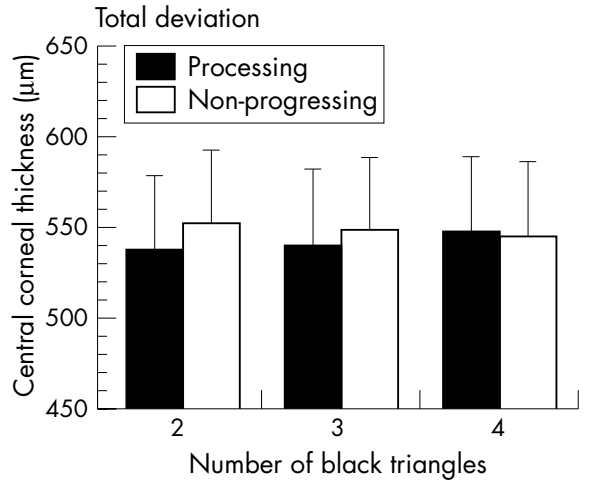

Number of black triangles

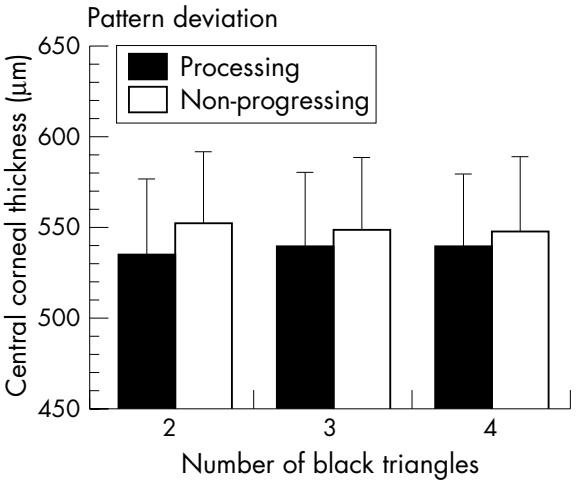

Figure 3 Mean central corneal thickness in eyes classified as progressing and non-progressing using increasingly conservative criteria (from two to four overlapping black triangles in three consecutive tests) with the glaucoma change probability analysis based on total deviation (left) and pattern deviation (right). Error bar $=1$ standard deviation. 


\begin{tabular}{|c|c|c|c|}
\hline \multirow[b]{2}{*}{ Index of change } & \multicolumn{3}{|l|}{$\mathrm{CCT}$ group } \\
\hline & $<524 \mu \mathrm{m}$ & $524-567 \mu \mathrm{m}$ & $>567 \mu \mathrm{m}$ \\
\hline EOC score (visual field) & $4.5(4.0)$ & $4.2(4.7)$ & $2.6(4.3)$ \\
\hline EOC score (optic disc) & $4.1(5.3)$ & $5.0(5.7)$ & $5.0(6.3)$ \\
\hline $\begin{array}{l}\text { Maximum number of black triangles (total } \\
\text { deviation) }\end{array}$ & $2.9(3.2)$ & $3.2(6.0)$ & $4.1(7.9)$ \\
\hline $\begin{array}{l}\text { Maximum number of black triangles (pattern } \\
\text { deviation) }\end{array}$ & $2.3(2.3)$ & $2.3(4.8)$ & $2.6(5.9)$ \\
\hline
\end{tabular}

probably have been treated more aggressively to attain lower IOP compared to those who did not progress, resulting in a wider range and variance of IOP during the follow up. This association therefore is probably an effect rather than a cause of visual field progression, especially since we did not show a similar relation between visual field progression and mean IOP. Interestingly none of the investigated factors were associated with optic disc progression. We used both eyes of most patients in the descriptive and statistical analysis; however, no adjustments to the probability levels (caused by inter-eye correlations) required for significance were made. Such adjustments make the statistical tests even more conservative suggesting that they may not have been useful in our study in which most of the descriptive trends relating to CCT and progression were either weak or inconsistent. The clinical or practical relevance of such observations would remain unaltered in spite of such adjustments.

The OHTS showed CCT to be a risk factor for the development of glaucoma. ${ }^{5}$ Assuming that there are CCT effects in addition to the sometimes inaccurate risk attributed to IOP, there are no compelling biological reasons to suggest that the risk attributed to these factors for developing glaucoma from ocular hypertension and for developing progression in eyes with clinically established damage should be substantially different. It is entirely possible that some subjects with ocular hypertension have already sustained damage and that the clinical diagnosis of glaucoma is limited by current diagnostic tests. A change from normal to abnormal with a less sensitive test could be interpreted as progression of existing damage with a more sensitive test. ${ }^{26}$

The distribution of CCT in the OHTS subjects ${ }^{27}$ was probably different from that of normal population, ${ }^{1}$ or a glaucoma sample drawn from a population study, containing a skew towards higher values. The mean CCT in identically divided subjects in our study was lower than that for the respective groups in the OHTS (by 19-28 $\mu \mathrm{m}$ ) and although the mean CCT of the group converting to glaucoma was similar to the overall mean in the present study $(553 \mu \mathrm{m}$ and $547 \mu \mathrm{m}$ respectively), those not converting to glaucoma had a higher mean CCT $(574 \mu \mathrm{m})$. Those subjects with ocular hypertension with substantially higher than average CCT would be at a considerably lower risk probably because their actual IOP would be within normal limits. When such individuals are combined with those with normal or below normal CCT and similar measured IOP (but higher actual IOP compared to those with higher CCT), the overall risk attributed to CCT may have been inflated. However, the OHTS demonstrated that within each similarly divided group based on IOP, thinner corneas were still associated with a higher conversion rate to glaucoma. Interestingly, the mean CCT of patients in the Early Manifest Glaucoma Treatment Study, which also did not find CCT to be a predictive factor for progressive glaucoma, was almost identical to that in our study. ${ }^{28}$

Although our study was prospective, it was non-interventional with respect to treatment regime. Patients were treated according to the visual field and optic disc findings generated from the study, in addition to the numerous other eye and patient related variables. CCT was measured towards the end of the follow up and therefore it is highly unlikely that knowledge of CCT and its possible influence on treatment patterns would have affected the outcome of this study. While in this case knowledge of CCT would not be a confounding factor, inappropriate target IOP (not low enough in an eye with a thin cornea or not high enough in an eye with a thick cornea), as a result of inaccurate IOP estimates, may have an effect on progression rates. While this was not apparent in our study, it was discussed as a possibility in a recent study which found an association between thin corneas and visual field progression. ${ }^{29}$

While our data did not find an association between CCT and glaucomatous field or disc progression, they do not rule out the importance of scleral or laminar thickness in glaucoma or the search for a reliable non-invasive method for its measurement. A measurement of CCT may be a poor correlate of posterior scleral thickness. Indeed, there is even uncertainty regarding an association between CCT and anterior scleral thickness. A recent study found a high correlation between CCT and scleral thickness at the scleral spur, but the relation was poorer, $1 \mathrm{~mm}$ and $2 \mathrm{~mm}$ posteriorly. ${ }^{30}$ Evidence from experimental myopia suggests that acquired changes in scleral thickness occur primarily at the

Table 2 Significance values from the multivariate regression analysis of the four outcome variables used for visual field and optic disc change

\begin{tabular}{|c|c|c|c|c|c|}
\hline \multirow[b]{2}{*}{ Dependent } & \multicolumn{5}{|c|}{ Independent } \\
\hline & $\begin{array}{l}\text { Baseline } \\
\text { MD }\end{array}$ & $\begin{array}{l}\text { Baseline } \\
\text { PSD }\end{array}$ & $\begin{array}{l}\text { Mean } \\
\text { IOP }\end{array}$ & $\begin{array}{l}\text { SD } \\
\text { IOP }\end{array}$ & СCT \\
\hline EOC score (visual field) & 0.159 & 0.035 & 0.108 & 0.001 & 0.922 \\
\hline EOC score (optic disc) & 0.194 & 0.113 & 0.088 & 0.411 & 0.762 \\
\hline $\begin{array}{l}\text { Maximum number of black triangles (total } \\
\text { deviation) }\end{array}$ & 0.212 & 0.029 & 0.051 & 0.001 & 0.022 \\
\hline $\begin{array}{l}\text { Maximum number of black triangles (pattern } \\
\text { deviation) }\end{array}$ & 0.442 & 0.028 & 0.309 & 0.006 & 0.067 \\
\hline
\end{tabular}


posterior pole $\mathrm{e}^{19}$ and therefore thickness estimates of the posterior sclera based on measurements of the anterior sclera or cornea may be inaccurate. From a practical perspective, a measurement of CCT can be valuable in the initial assessment of the glaucoma suspect, particularly in ruling out a high risk suggested by an elevated IOP in an eye with high CCT but normal optic disc and visual field findings. Once glaucomatous damage is present, CCT is unlikely to be a useful predictive index for visual field and optic disc progression, even when there is a large range of CCT in the glaucoma population such as that in this study.

\section{ACKNOWLEDGEMENTS}

This study was supported by grant MOP-11357 from the Canadian Institutes of Health Research (BCC and RPL).

\section{Authors' affiliations}

B C Chauhan, D M Hutchison, R P LeBlanc, P H Artes, M T Nicolela, Department of Ophthalmology and Visual Sciences, Dalhousie University, Halifax, NS, Canada

B C Chauhan, Department of Physiology and Biophysics, Dalhousie University, Halifax, NS, Canada

Ethical approval: The study was approved by the Queen Elizabeth II Health Science Centre Research Ethics Committee. All patients gave written informed consent.

\section{REFERENCES}

1 Doughty MJ, Zaman ML. Human corneal thickness and its impact on intraocular pressure measures: a review and meta-analysis approach. Surv Ophthalmol 2000;44:367-408.

2 Ehlers N, Bramsen T, Sperling S. Applanation tonometry and central corneal thickness. Acta Ophthalmol (Copenh) 1975;53:34-43.

3 Whitacre MM, Stein RA, Hassanein K. The effect of corneal thickness on applanation tonometry. Am J Ophthalmol 1993;115:592-6.

4 Johnson M, Kass MA, Moses RA, et al. Increased corneal thickness simulating elevated intraocular pressure. Arch Ophthalmol 1978;96:664-5.

5 Gordon MO, Beiser JA, Brandt JD, et al. The Ocular Hypertension Treatment Study: baseline factors that predict the onset of primary open-angle glaucoma. Arch Ophthalmol 2002;120:714-20; discussion 829-30.

6 Medeiros FA, Sample PA, Weinreb RN. Corneal thickness measurements and visual function abnormalities in ocular hypertensive patients. Am J Ophthalmol 2003;135:131-7.

7 Medeiros FA, Sample PA, Weinreb RN. Corneal thickness measurements and frequency doubling technology perimetry abnormalities in ocular hypertensive eyes. Ophthalmology 2003;110:1903-8.

8 Anderson DR, Hendrickson A. Effect of intraocular pressure on rapid axoplasmic transport in monkey optic nerve. Invest Ophthalmol 1974;13:771-83

9 Minckler DS, Bunt AH, Johanson GW. Orthograde and retrograde axoplasmic transport during acute ocular hypertension in the monkey. Invest Ophthalmol Vis Sci 1977;16:426-41.
10 Gaasterland D, Tanishima T, Kuwabara T. Axoplasmic flow during chronic experimental glaucoma. 1. Light and electron microscopic studies of the monkey optic nervehead during development of glaucomatous cupping. Invest Ophthalmol Vis Sci 1978;17:838-46.

11 Radius RL, Bade B. Axonal transport interruption and anatomy at the lamina cribrosa. Arch Ophthalmol 1982;100:1661-4

12 Pease ME, McKinnon SJ, Quigley HA, et al. Obstructed axonal transport of BDNF and its receptor TrkB in experimental glaucoma. Invest Ophthalmol Vis Sci 2000:41:764-74.

13 Morgan WH, Yu DY, Cooper RL, et al. The influence of cerebrospinal fluid pressure on the lamina cribrosa tissue pressure gradient. Invest Ophthalmol Vis Sci 1995;36:1163-72.

14 Artes PH, Chauhan BC. Longitudinal changes in the visual field and optic disc in glaucoma. Prog Retin Eye Res 2005;24:333-54.

15 Junemann AG, Martus P, Wisse M, et al. Quantitative analysis of visual field and optic disk in glaucoma: retinal nerve fiber bundle-associated analysis. Graefes Arch Clin Exp Ophthalmol 2000;238:306-14

16 Heijl A, Lindgren G, Lindgren A, et al. Extended empirical statistical package for evaluation of single and multiple fields: Statpac 2. New York: Kugler and Ghedini, 1991.

17 Downs JC, Ensor ME, Bellezza AJ, et al. Posterior scleral thickness in perfusion-fixed normal and early-glaucoma monkey eyes. Invest Ophthalmol Vis Sci 2001;42:3202-8.

18 Curtin BJ, Teng CC. Scleral changes in pathological myopia. Trans Am Acad Ophthalmol Otolaryngol 1958;62:777-88; discussion 788-90.

19 McBrien NA, Cornell LM, Gentle A. Structural and ultrastructural changes to the sclera in a mammalian model of high myopia. Invest Ophthalmol Vis Sci 2001;42:2179-87.

20 McBrien NA, Gentle A. Role of the sclera in the development and pathological complications of myopia. Prog Retin Eye Res 2003;22:307-38.

21 Mitchell P, Hourihan F, Sandbach J, et al. The relationship between glaucoma and myopia: the Blue Mountains Eye Study. Ophthalmology 1999; 106:2010-15.

22 Jonas JB, Berenshtein E, Holbach L. Lamina cribrosa thickness and spatial relationships between intraocular space and cerebrospinal fluid space in highly myopic eyes. Invest Ophthalmol Vis Sci 2004;45:2660-5.

23 Martinez-Bello C, Chauhan BC, Nicolela MT, et al. Intraocular pressure and progression of glaucomatous visual field loss. Am J Ophthalmol 2000; 129:302-8

24 Stewart WC, Kolker AE, Sharpe ED, et al. Factors associated with long-term progression or stability in primary open-angle glaucoma. Am J Ophthalmol 2000;130:274-9.

25 Nouri-Mahdavi K, Hoffman D, Coleman AL, et al. Predictive factors for glaucomatous visual field progression in the Advanced Glaucoma Intervention Study. Ophthalmology 2004;111:1627-35.

26 Johnson CA, Adams AJ, Casson EJ, et al. Blue-on-yellow perimetry can predict the development of glaucomatous visual field loss. Arch Ophthalmol 1993; 111:645-650.

27 Brandt JD, Beiser JA, Kass MA, et al. Central corneal thickness in the Ocular Hypertension Treatment Study (OHTS). Ophthalmology 2001;108:1779-88.

28 Leske MC, Heijl A, Hussein M, et al. Factors for glaucoma progression and the effect of treatment: the early manifest glaucoma trial. Arch Ophthalmol 2003; 121:48-56

29 Kim JW, Chen PP. Central corneal pachymetry and visual field progression in patients with open-angle glaucoma. Ophthalmology 2004;111:2126-32.

30 Oliveira C, Tello C, Ritch R, et al. Correlation between central corneal thickness, scleral thickness and refractive error [abstract]. Annual meeting of the Association for Research in Vision and Ophthalmology, 2004:963. 